\title{
An Insight in Understanding the Symptomatology of $\beta$-Thalassaemia Major - II
}

Most of the symptoms of $\beta$-thalassaemia major are considered to be due to anaemia and massive iron overload in the body [1]. Despite of regular blood transfusion and iron chelation, delayed growth, risk of infection \& thrombosis, asthenia, and exercise intolerance remain a major reason of debility for patients, and a major concern for treating physicians [1]. Treatment with Hydroxyurea (HU) in $\beta$-thalassaemia major and intermedia has ameliorated the need of blood transfusion [2-6]. One consistent finding in these patients is subjective improvement (as reported by almost mothers of these kids) in quality of life, vitality, better exercise tolerance, improved food intake and energy level within a couple of weeks of exposure to the drug despite of minimal or no change in haemoglobin level [2-6]. This improvement persisted during the course of treatment even in partial responders (who were maintaining an $\mathrm{Hb}$ of 6-7 g/dl). Recently, additional benefit of $\mathrm{HU}$ on iron chelation/reduction in serum ferritin has been reported $[7,8]$.

How exactly does HU work in $\beta$-thalassaemia is poorly understood. Effects of HU at sub-cellular level i.e. on intracellular anti-oxidant levels, metabolic pathways, handling of trace elements required for enzymes in different metabolic pathways, oxygen binding and dissociation from haemoglobin-F are not entirely known. Our group has previously reported interesting results of proteomics, metallomics, metabolomics and genomics in $\beta$-thalassemia patients with and without the institution of HU [9-13]. Primary and secondary genetic modifiers, like beta globin gene mutations and presence or absence of XMN-1, HSB1L-MYB, and BCL-11A polymorphisms, have been found to significantly influence the response of HU in improving haemoglobin levels in beta thalassemia patients [14].

Excessive $\alpha$-globin chains in red cells cause irreversible oxidative damage to inner part of red cell membrane lipids and proteins by hemichromes formation and increasing intracellular calcium concentration, resulting in severe haemolytic anaemia [6]. Prolonged severe anaemia stimulates erythropoietic activity culminating in hepatosplenomegaly due to extramedullary erythropoiesis. It further causes iron overload induced dysfunctions of various organs, thrombosis, diabetes mellitus, severe infections, and growth retardation [7].

It has recently been reported that the Metallomic profile of $\beta$-thalassaemia patients is markedly deranged. After commencement of HU, Eight out of nineteen analyzed elements, in the serum of $\beta$-thalassemia patients, were shown to be corrected. HU not only improved $\mathrm{Hb}$ levels in these patients but also reduced metal dysregulations resulting in normalization of many metallomic pathways [9]. As these metals function as important cofactors and also as part of many enzymes at sub-cellular level, their dysregulation explains some of the symptomatology of this disease. This HU induced normalization effect at metallomics level possibly translates into improved quality of life and exercise tolerance in $\beta$-thalassaemia patients [13].

Studying metabolome of $\beta$-thalassemia patients, to understand unclear pathophysiological mechanisms of thalassemia and for prognostic evaluation, started to unveil many interesting and important differences in serum metabolites of $\beta$-thalassemia patients and normal subjects. Many studies have shown that dysregulation of metabolomic profile is common in $\beta$-thalassemia patients. Studies of the biomarkers for lipid peroxidation-induced DNA damage, plasma substance $\mathrm{P}$ and soluble P-selectin as indicator of hypercoagulability in these patients, adipo-cytokines related to haemolytic and inflammatory biomarkers, biomarkers of iron and oxidant-antioxidant homeostasis have provided important information to comprehend the symptomatology of $\beta$-thalassaemia major and intermedia.

Recently, metabolic pathway analysis has reported alterations in fatty acid, glycolytic, galactose, pyruvate, propanoate, glycerophospholipid, and sucrose metabolism along with fatty acid elongation in the mitochondria, glycerolipid, glyoxylate and dicarboxylate metabolism pointing towards a major metabolic shift in $\beta$-thalassemia patients compared to healthy individuals [13]. Follow up studies in these patients after exposure to HU revealed reversal of many of these metabolic pathways towards normal. This sheds some light on how asthenia, growth retardation, and many other body functions are compromised in thalassaemia syndrome.

Our group also performed proteomics studies in pre- and post- HU-treated $\beta$-thalassemia patients, and compared the results in responders and non-responders to HU treatment. One important initial finding was that serum paraoxonase activity and malondialdehyde serum concentrations remain unaffected in response to hydroxyurea therapy in $\beta$-thalassemia patients [11]. This implies that HU does not adversely affect metabolic pathways in these patients. Extension of this work revealed that twenty-one proteins were found to be significantly different in pre- vs post- HU treated groups (unpublished data). Interesting finding is reversal of dysregulated proteins towards normal in HU treated patients.

Studies on sub-cellular pathways have started to provide important information on the pathophysiology of thalassaemia syndrome. Haemoglobin-F augmentation utilizing HU is now better understood than ever before. There still are many important 
questions which remained unanswered. Many in-depth studies are needed to extract all the answers.

\section{REFERENCES}

[1] Weatherall DJ, Clegg JB. The Thalassemia Syndromes. 4th ed. Oxford: Blackwell Science 2001; pp. 288-9. DOI: $10.1002 / 9780470696705$

[2] Perrine SP. Fetal globin induction-can it cure $\beta$-thalassemia? Hematol Am Soc Hematol Educ Prog 2005; 38-44.

[3] Ansari SH, Shamsi TS, Ashraf M, et al. Efficacy of hydroxyurea in providing transfusion independence in $\beta$-thalassemia. J Pediatr Hematol Oncol 2011; 33: 339-43. DOI: 10.1097/MPH.0b013e31821b0770

[4] Bradai M, Abad MT, Pissard S, Lamraoui F, Skopinski L, de Montalembert M. Hydroxyurea can eliminate transfusion requirements in children with severe $\beta$-thalassemia. Blood 2003; 104: 114-7. DOI: 10.1182/blood-2003-01-0117

[5] Zamani F, Shakeri R, Eslami SM, Razavi SM, Basi A. Hydroxyurea therapy in 49 patients with major $\beta$-thalassemia. Arch Iran Med 2009; 12(3): 295-7.

[6] Yavarian M, Karimi M, Bakker E, Harteveld CL, Giordano PC. Response to hydroxyurea treatment in Iranian transfusion-dependent $\beta$-thalassemia patients. Haematologica 2004; 89: 1172-8.

[7] Dixit A, Chatterjee TC, Mishra P. Hydroxyurea in thalassemia intermedia-a promising therapy. Ann Hematol 2005; 84: 441-6. DOI: $10.1007 / \mathrm{s} 00277-005-1026-4$

[8] Italia K, Colah R, Ghosh K. Hydroxyurea could be a good clinically relevant iron chelator. PLoS One 2013; 8: e82928. 31.

[9] Giardina PJ, Grady RW. Chelation therapy in $\beta$-thalassemia: An optimistic update. Semin Hematol 2001; 38: 360-6. DOI: 10.1016/S0037-1963(01)90030-7

[10] Mazhar W, Farooq S, Iqbal A, et al. Metallomic profiling to evaluate the response to drug treatment: Hydroxyurea as a case study in $\beta$-thalassemia patients. RSC Adv 2017; 7: 23882-9. DOI: 10.1039/C6RA28514G

[11] Zohaib M, Ansari SH, Hashim Z, Shamsi TS, Zarina S. Serum paraoxonase activity and malondialdehyde serum concentrations remain unaffected in response to hydroxyurea therapy in $\beta$-thalassemia patients. J Clin Pharmacol 2016; 56(7): 869-74. DOI: 10.1002/jcph.675

[12] Ansari SH, Shamsi TS, Ashraf M, et al. Molecular epidemiology of $\beta$-thalassemia in Pakistan: Far reaching implications. Int J Mol Epidemiol Genet 2011; 2(4): 403-8.

[13] Musharraf SG, Iqbal A, Ansari SH, Parveen S, Khan IA, Siddiqui AJ. $\beta$ - thalassemia patients revealed a significant change of untargeted metabolites in comparison to healthy individuals. Sci Rep 2017; 7: 42249. DOI: 10.1038/srep42249

[14] Rujito L, Basalamah M, Siswandari W, et al. Modifying effect of XmnI, BCL11A, and HBS1L-MYB on clinical appearances: A study on $\beta$-thalassemia and hemoglobin E/ $\beta$-thalassemia patients in Indonesia. Hematol Oncol Stem Cell Ther 2016; 9(2): 55-63.

DOI: $10.1016 /$ j.hemonc.2016.02.003

\section{Editor-in-Chief}

Tahir Shamsi

National Institute of Blood Disease \& Bone Marrow Transplantation, Karachi, Pakistan 